\title{
ECLETICA
}

www.scielo.br/eq

Volume 31, número 1, 2006

\section{Uso da análise exploratória de dados na avaliação de modificadores químicos para determinação direta e simultânea de metais em álcool combustível por GFAAS}

\author{
A. P. de Oliveira ${ }^{1 *}$, J. A. Gomes Neto ${ }^{1}$, M. M. C. Ferreira ${ }^{2}$ \\ ${ }^{\prime}$ Instituto de Química - Universidade Estadual Paulista, UNESP, CP 355, CEP: 14801-970 Araraquara - SP. \\ ${ }^{2}$ Instituto de Química - Universidade Estadual de Campinas, UNICAMP, CP 6154, CEP:13084-971 Campinas - SP. \\ *dri_poliv@ig.com.br
}

\begin{abstract}
Resumo: O objetivo deste trabalho foi empregar a análise exploratória de dados, no caso, a técnica de análise de componentes principais (PCA) como ferramenta na avaliação de modificadores químicos na determinação direta e simultânea de $\mathrm{Al}, \mathrm{As}, \mathrm{Cu}, \mathrm{Fe}$, Mn e Ni em álcool etílico combustível por espectrometria de absorção atômica em forno de grafite (GFAAS). Os modificadores químicos avaliados foram: $\mathrm{Pd}\left(\mathrm{NO}_{3}\right)_{2}+\mathrm{Mg}\left(\mathrm{NO}_{3}\right)_{2}$; W/Rh; W+ co-injeção de $\mathrm{Pd}\left(\mathrm{NO}_{3}\right)_{2}+\mathrm{Mg}\left(\mathrm{NO}_{3}\right)_{2}$ e para cada modificador foram utilizadas trintas amostras de álcool etílico combustível. Como dados experimentais foram utilizados os resultados dos testes de adição e recuperação dos analitos frente aos diferentes modificadores químicos estudados. O emprego da técnica de PCA possibilitou a separação dos tipos de modificadores em função do intervalo de recuperação do analito. Dentre os modificadores avaliados, W+ co-injeção de $\mathrm{Pd}\left(\mathrm{NO}_{3}\right)_{2}+\mathrm{Mg}\left(\mathrm{NO}_{3}\right)_{2}$ apresentou-se como a espécie de maior correlação positiva, pois apresenta os maiores teores de recuperação, e sendo assim, foi o escolhido para o desenvolvimento de metodologia para determinação direta e simultânea de $\mathrm{Al}, \mathrm{As}, \mathrm{Cu}, \mathrm{Fe}, \mathrm{Mn}$ e Ni em álcool etílico combustível por GFAAS.
\end{abstract}

Palavras-chave: Análise exploratória de dados; Quimiometria; PCA; GFAAS; álcool combustível.

\section{Introdução}

O álcool etílico é considerado um combustível alternativo e altamente viável. A sua produção é obtida através da biomassa, caracterizando-o assim como um combustível renovável e limpo [1-3].

O monitoramento quantitativo de elementos metálicos em combustíveis é de relevância econômica para a indústria de combustíveis assim como para as áreas automobilísticas e ambientais. Por meio de um controle analítico eficiente dos teores de aditivos inorgânicos incidentais ou acidentais, é possível gerenciar, desde a produção até os pontos de distribuição, a qualidade dos combustíveis oferecidos ao consumidor. Devido aos baixos teores dos metais usualmente presentes no álcool etílico hidratado combustível, técnicas analíticas sensíveis são requeridas tal como a espectrometria de absorção atômica em forno de grafite (GFAAS) [1].

O emprego de modificadores químicos é uma prática comum em espectrometria de absorção atômica com atomização eletrotérmica em forno de grafite. A sua principal função é diminuir, ou até mesmo eliminar, interferências provocadas pela matriz ou concomitantes presentes na amostra, por meio do aumento da efi- 
ciência da etapa de pirólise. O principal objetivo da utilização dos modificadores químicos é permitir altas temperaturas de pirólise. Com uma temperatura de pirólise elevada, é viável a eliminação dos concomitantes, sem com isso, afetar a atomização do analito de interesse. O modificador químico converte o analito em uma forma menos volátil, permitindo maiores temperaturas de pirólise e/ou converte os concomitantes em uma forma mais volátil, promovendo a separação analito-matriz [4]. A seleção do modificador químico ótimo é um parâmetro crítico para determinações diretas e simultâneas em GFAAS e o uso da Quimiometria, permite a visualização em gráficos da tabela de resultados analíticos, relacionando as amostras e as variáveis selecionadas.

Em diversos segmentos da química o crescimento do uso de recursos computacionais para analisar dados químicos cresceu nos últimos anos. $\mathrm{Na}$ área de química analítica a aquisição de dados atingiu um ponto bastante sofisticado, devido ao aumento da quantidade dos dados analíticos gerados e ao interfaceamento dos instrumentos aos computadores produzindo uma enorme quantidade de informação, muitas vezes complexa e variada [5].

A necessidade de ferramentas novas para extrair um maior número informações relevantes de um conjunto de dados deu origem a Quimiometria, que é uma área destinada especificamente à análise de dados químicos de natureza multivariada. Os métodos multivariados consideram a correlação entre muitas variáveis analisadas simultaneamente, permitindo a extração de uma quantidade maior de informações, muitas vezes impossível quando se analisa variável a variável [6].

A análise de componentes principais (PCA) é um método de análise multivariada utilizado para projetar dados n-dimensionais em um espaço de baixa dimensão, normalmente duas ou três. Isso é feito através do cálculo de componentes principais obtidas fazendo-se combinações lineares das variáveis originais. PCA é um método exploratório porque auxilia na elaboração de hipóteses gerais a partir dos dados coletados, contrastando com estudos direcionados nos quais hipóteses prévias são testadas. É também capaz de separar a informação importante da redundante e aleatória. Em uma análise de componentes principais, o agrupamento das amostras define a estrutura dos dados através de gráficos de scores e loadings, cujos eixos são componentes principais (PCs) nos quais os dados são projetados. Os scores fornecem a composição das PCs em relação às amostras, enquanto os loadings fornecem essa mesma composição em relação às variáveis. Como as PCs são ortogonais, é possível examinar as relações entre amostras e variáveis através dos gráficos dos scores e dos loadings. O estudo conjunto de scores e loadings ainda permite estimar a influência de cada variável em cada amostra [7-11].

O objetivo deste trabalho é empregar a análise exploratória de dados (PCA), como uma ferramenta na avaliação de modificadores químicos na determinação direta e simultânea de $\mathrm{Al}, \mathrm{As}, \mathrm{Cu}, \mathrm{Fe}, \mathrm{Mn}$ e Ni em álcool combustível por GFAAS. Os modificadores químicos avaliados foram: $\mathrm{Pd}\left(\mathrm{NO}_{3}\right)_{2}+\mathrm{Mg}\left(\mathrm{NO}_{3}\right)_{2}$; $\mathrm{W} / \mathrm{Rh} ; \mathrm{W}+$ co injeção de $\mathrm{Pd}\left(\mathrm{NO}_{3}\right)_{2}+$ $\mathrm{Mg}\left(\mathrm{NO}_{3}\right)_{2}$, e para cada modificador foram utilizadas trintas amostras de álcool etílico combustível. Estes modificadores químicos foram previamente selecionados, devido à presença de trabalhos na literatura relacionados aos analitos de interesse. Como dados experimentais foram utilizados os resultados dos testes de adição e recuperação dos analitos frente aos diferentes modificadores químicos estudados.

\section{Procedimento Experimental}

\section{Instrumentos e acessórios}

$\mathrm{O}$ instrumento utilizado foi um espectrômetro de absorção atômica Perkin-Elmer, Modelo SIMAA 6000, equipado com amostrador automático Perkin-Elmer, Modelo AS-72, corretor de fundo por efeito Zeeman longitudinal e tubo de grafite com aquecimento transversal (THGA) e plataforma de L'vov integrada. Como fontes de radiação foram utilizadas as seguintes lâmpadas: lâmpada de descarga sem eletrodo (EDL) de As $(\lambda=193,7$ nm e i $=300$ mA); lâmpada de catodo oco multielementar para $\mathrm{Al}$ e $\mathrm{Fe}$ $(\lambda=309,3 \mathrm{~nm}(\mathrm{Al})$ e $\lambda=248,3 \mathrm{~nm}(\mathrm{Fe})$ e $\mathrm{i}=30$ $\mathrm{mA})$; lâmpada de catado oco de $\mathrm{Ni}(\lambda=230,0 \mathrm{~nm}$ e i= $25 \mathrm{~mA}$ ) ; lâmpada de catodo oco multiele- 
mentar de $\mathrm{Cu}$ e $\mathrm{Mn}(\lambda=324,8 \mathrm{~nm}(\mathrm{Cu})$ e $\lambda=279,5$ $\mathrm{nm}(\mathrm{Mn})$ e $\mathrm{i}=20 \mathrm{~mA})$. A largura da fenda foi de 0,7 nm e o fluxo de Argônio (99.999\%, White Martins) foi de $250 \mathrm{~mL} \cdot \mathrm{min}^{-1}$.

\section{Reagentes, soluções e amostras}

Todas as soluções foram preparadas utilizando-se água desionizada de alta pureza (resistividade 18,2 M $\Omega . c m$ ) obtida em sistema Milli-Q, Millipore.

Ácido Nítrico Suprapur ${ }^{\circledR}$, Merck, foi utilizado para preparar as soluções em meio ácido.

Solução estoque de arsênio (1000 $\left.\mathrm{mg} \mathrm{L}^{-1}\right)$ foi preparada pela dissolução de $1,320 \mathrm{~g} \mathrm{As}_{2} \mathrm{O}_{3}$ (Fluka Chemical) em $20 \mathrm{~mL}$ de solução de $\mathrm{NaOH}$ $1,0 \mathrm{~mol} \mathrm{~L}^{-1}$. Um volume de $50 \mathrm{~mL}$ de uma solução de $\mathrm{HCl} 0,5 \mathrm{~mol} \mathrm{~L}^{-1}$ foi adicionado a essa solução e o volume foi completado para $1000 \mathrm{~mL}$ com água. As soluções estoque de cobre, manganês, ferro e níquel (1000 $\left.\mathrm{mg} \mathrm{L}^{-1}\right)$ foram preparados a partir dos respectivos padrões para absorção atômica, Normex ${ }^{\circledR}$, Carlo Erba. Solução estoque de alumínio (1000 $\left.\mathrm{mg} \mathrm{L}^{-1}\right)$ foi preparada a partir do padrão para absorção atômica Titrisol ${ }^{\circledR}$, Merck.

Solução de modificador químico (1000 mg L-1 Pd) foi preparada a partir da solução estoque $10000 \mathrm{mg} \mathrm{L}^{-1} \mathrm{Pd}\left(\mathrm{NO}_{3}\right)_{2}$ em $15 \% \mathrm{HNO}_{3}$, (Perkin-Elmer, Part № BO190635). Solução de modificador químico (1000 $\mathrm{mg} \mathrm{L}^{-1} \mathrm{Mg}$ ) foi preparada a partir da solução estoque $10000 \mathrm{mg}$ $\mathrm{L}^{-1} \quad \mathrm{Mg}\left(\mathrm{NO}_{3}\right)_{2} \quad$ (Perkin-Elmer, Part № BO190634). Para o modificador químico permanente $\mathrm{W} / \mathrm{Rh}$, a solução de $1000 \mathrm{mg} \mathrm{L}^{-1} \mathrm{~W}$ foi preparada pela dissolução de $0,1794 \mathrm{~g}$ de $\mathrm{NaWO}_{4} \cdot 2 \mathrm{H}_{2} \mathrm{O}$ (Merck) em $100 \mathrm{~mL}$ de água desionizada e a solução de $1000 \mathrm{mg} \mathrm{L}^{-1} \mathrm{Rh}$ foi preparada pela dissolução de $51 \mathrm{mg}$ de $\mathrm{RhCl}_{3}$ (Sigma,USA) em $25 \mathrm{~mL}$ de solução $2 \%$ (v/v) $\mathrm{HNO}_{3}{ }^{21}$.

Soluções analíticas de referência multielementares de $\mathrm{Al}, \mathrm{As}, \mathrm{Cu}, \mathrm{Fe}, \mathrm{Mn}$ e $\mathrm{Ni}$ foram preparadas diariamente por diluições sucessivas das respectivas soluções estoque monoelementares.

As amostras de álcool etílico hidratado combustível foram coletadas em diferentes postos de abastecimento da cidade de Araraquara-SP.

\section{Análise Química}

Para a verificar a exatidão de um método pode-se empregar o uso de materiais de referência certificados, teste de adição e recuperação do analito e a comparação dos resultados com técnica ou método alternativo. Neste trabalho foi empregado o teste de adição e recuperação dos analitos.

Determinações multielementares de As, $\mathrm{Al}, \mathrm{Cu}, \mathrm{Fe}, \mathrm{Mn}$ e Ni foram feitas em 30 amostras álcool etílico combustível contendo $60,0 \mu \mathrm{g} \mathrm{L} \mathrm{L}^{-1}$ $\mathrm{Al}, \mathrm{As}, \mathrm{Cu}, \mathrm{Fe}, \mathrm{Mn}$ e Ni (1+1; v/v) em 2,0\% (v/v) $\mathrm{HNO}_{3}$. Como brancos analíticos, foram feitas determinações em 30 amostras de álcool etílico combustível (1+1; v/v) em 2,0\% (v/v) $\mathrm{HNO}_{3}$. Os sinais transientes de absorção atômica e de fundo dos seis elementos foram medidos simultaneamente.

Este procedimento foi realizado nos diferentes modificadores químicos: $\mathrm{Pd}\left(\mathrm{NO}_{3}\right)_{2}+$ $\mathrm{Mg}\left(\mathrm{NO}_{3}\right)_{2}$; W/Rh; W+ co-injeção de $\mathrm{Pd}\left(\mathrm{NO}_{3}\right)_{2}+$ $\mathrm{Mg}\left(\mathrm{NO}_{3}\right)_{2}$.

\section{Análise multivariada}

A técnica de análise de componentes principais (PCA) foi utilizada correlacionando os intervalos de recuperação obtidos no teste de adição e recuperação para cada elemento nos diferentes modificadores químicos, com o intuito de avaliar e selecionar o modificador químico ótimo para o desenvolvimento de metodologia para determinação direta e simultânea $\mathrm{As}, \mathrm{Al}, \mathrm{Cu}, \mathrm{Fe}, \mathrm{Mn}$ e Ni em álcool etílico combustível por GFAAS.

O programa Pirouette (InfoMetrix, Woodinville, Washington, USA) versão 2.02 foi utilizado para efetivar as análises deste trabalho por oferecer um ambiente computacional amplamente utilizado em quimiometria e diversas outras áreas $[8,9]$.

O auto-escalamento foi o pré-processamento utilizado no tratamento dos dados em função das diferentes ordens de grandeza das variáveis estudadas, atribuindo-se assim um mesmo peso para todas as variáveis.

\section{Resultados e discussão}

A necessidade da utilização de uma ferra- 
menta estatística para analisar e interpretar uma grande quantidade de informações foi comprovada por meio da observação do conjunto de dados obtidos nesta proposta de trabalho. Os seis analitos foram determinados simultaneamente em 30 amostras de álcool etílico combustível em três diferentes tipos de modificadores químicos e geraram uma matriz de dados $90 \times 6$, da qual poucas informações podem ser extraídas através da simples observação. A técnica da análise multivariada (PCA) foi aplicada visando resumir em poucas e importantes dimensões a maior parte da variabilidade da matriz para obtenção de relações entre os dados obtidos [10].

Um pré-processamento dos dados foi necessário para atribuir pesos equivalentes às percentagens de recuperação dos analitos nas amostras[11]. Assim, os dados foram auto-escalados antes de serem submetidos à análise de componentes principais.

A PCA mostrou que com apenas dois componentes principais é possível descrever $82,4 \%$ dos dados, sendo $63,5 \%$ da variância total descrita pela primeira componente principal (PC1) e 18,9\% por PC2.

Por meio do gráfico de scores (Figura 1), observou-se à formação de três agrupamentos, representando as espécies de modificadores químicos estudados. A PC1 com $63,5 \%$ da variância total, apresentou como variáveis positivas $\mathrm{W} / \mathrm{Rh}$ e $\mathrm{W}+$ co-injeção de $\mathrm{Pd}\left(\mathrm{NO}_{3}\right)_{2}+\mathrm{Mg}\left(\mathrm{NO}_{3}\right)_{2}$.
O gráfico de loadings (Figura 2) mostra que o elemento $\mathrm{Mn}$ apresentou melhores resultados de recuperação quando se utilizou o modificador químico $\mathrm{Pd}\left(\mathrm{NO}_{3}\right)_{2}+\mathrm{Mg}\left(\mathrm{NO}_{3}\right)_{2}$. No caso do elemento As, os melhores resultados foram para o modificador permanente $\mathrm{W} / \mathrm{Rh}$. Porém, para os demais elementos, o modificador $\mathrm{W}+$ coinjeção de $\mathrm{Pd}\left(\mathrm{NO}_{3}\right)_{2}+\mathrm{Mg}\left(\mathrm{NO}_{3}\right)_{2}$ apresentou-se com uma maior correlação positiva, ou seja, com melhores valores de recuperação.

Como neste trabalho estamos realizando determinações simultâneas, o modificador químico ótimo deve ser aquele que apresente melhores valores de recuperação para a maioria dos analitos de interesse, porém sem o comprometimento dos demais. Então, o modificador W+ co-injeção de $\mathrm{Pd}\left(\mathrm{NO}_{3}\right)_{2}+\mathrm{Mg}\left(\mathrm{NO}_{3}\right)_{2}$ se enquadra neste contexto, pois a perda da qualidade dos resultados para Mn e As em comparação aos outros modificadores foi de apenas 3,0\% [12].

Além disso, o modificador químico W+ co-injeção de $\operatorname{Pd}\left(\mathrm{NO}_{3}\right)_{2}+\operatorname{Mg}\left(\mathrm{NO}_{3}\right)_{2}$ apresentou melhores valores de limite de detecção (L.D), massa característica $\left(m_{O}\right)$ e desvio padrão relativo (R.S.D). Outro fator importante foi o aumento do tempo de vida útil do atomizador. Para o modificador químico W+ co-injeção de $\mathrm{Pd}\left(\mathrm{NO}_{3}\right)_{2}+$ $\mathrm{Mg}\left(\mathrm{NO}_{3}\right)_{2}$ o aumento foi de cerca de $25 \%$ em relação aos outros modificadores avaliados [12].

Como o presente trabalho tem como objetivo principal à determinação simultânea, o mo-

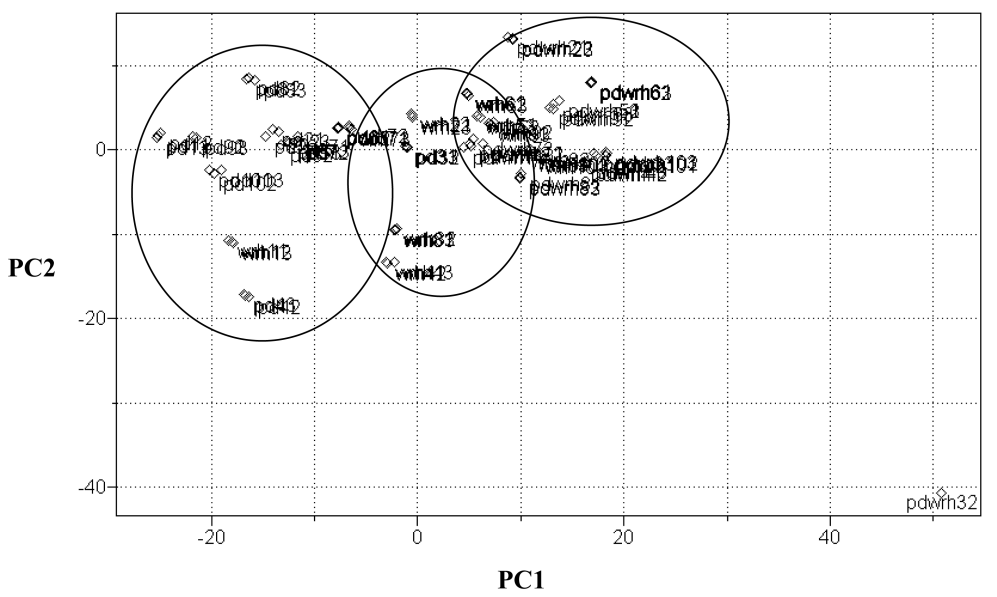

Figura 1. Análise por componentes principais: PC1x PC2. Gráfico de scores. 


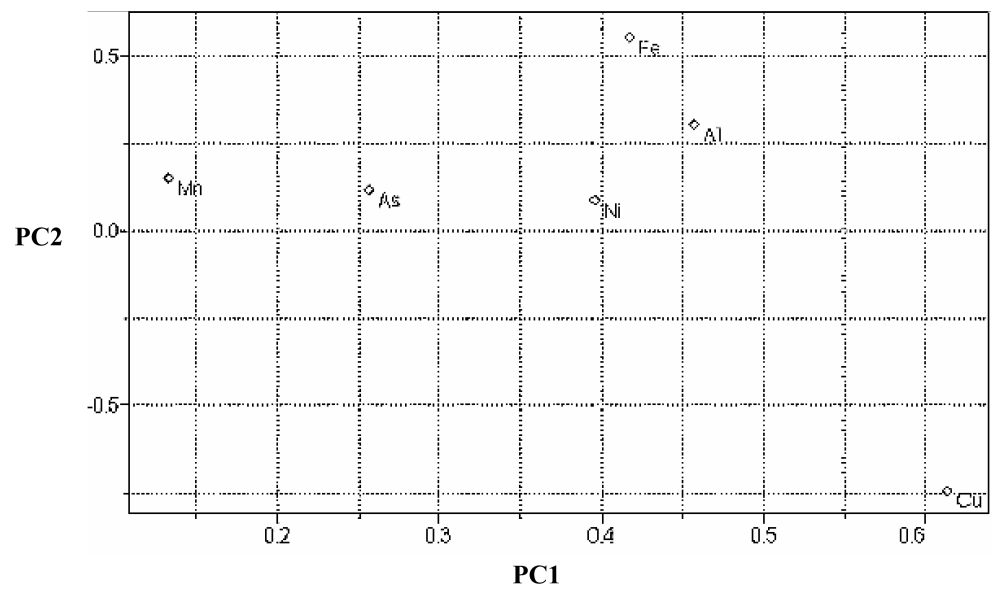

Figura 2. Análise por componentes principais: PC1x PC2. Gráfico de loadings.

dificador químico $\mathrm{W}+$ co-injeção de $\mathrm{Pd}\left(\mathrm{NO}_{3}\right)_{2}+$ $\mathrm{Mg}\left(\mathrm{NO}_{3}\right)_{2}$ foi o escolhido para o desenvolvimento de metodologia para determinação direta e simultânea As, Al, Cu, Fe, Mn e Ni em álcool etílico combustível por GFAAS.

\section{Conclusões}

Este trabalho ressalta a importância da utilização da quimiometria para o tratamento dos dados químicos. O emprego da análise por componentes principais (PCA) evidenciou as características comuns e discrepantes entre as diferentes amostras, importantes para o desenvolvimento de novas metodologias em GFAAS, porém dificilmente visualizadas diretamente na matriz de dados.

A PCA possibilitou extrair informações relevantes, tais como correlacionar os intervalos de recuperação obtidos pelo emprego do teste de adição e recuperação do analitos e os diferentes modificadores químicos.

Dentre os modificadores químicos avaliados, $\mathrm{W}+$ co-injeção de $\mathrm{Pd}+\mathrm{Mg}$ apresentou-se como a espécie de maior correlação positiva, pois apresentou os maiores teores de recuperação e sendo assim, foi selecionado como modificador químico para o desenvolvimento de metodologia para determinação direta e simultânea $\mathrm{As}, \mathrm{Al}, \mathrm{Cu}$, $\mathrm{Fe}, \mathrm{Mn}$ e Ni em álcool etílico combustível por espectrometria de absorção atômica em forno de grafite.

\section{Agradecimentos}

Os autores agradecem à FAPESP pelo suporte financeiro e pela bolsa concedida.

Recebido em: 24/10/2005

Aceito em: 15/12/2005 
A. P. de Oliveira, J. A. Gomes Neto, M. M.C. Ferreira. The use of the exploratory analysis in the evaluation of chemical modifiers for direct and simultaneous determination of metals in fuel ethanol by GFAAS.

Abstract: The aim of this work was to employ the exploratory analysis, in this case, the principal component analysis (PCA) as an alternative tool in the evaluation of chemical modifiers for direct and simultaneous determination of $\mathrm{Al}, \mathrm{As}, \mathrm{Cu}, \mathrm{Fe}, \mathrm{Mn}$ and $\mathrm{Ni}$ in ethanol fuel by graphite furnace atomic absorption spectrometry (GFAAS). The evaluated chemical modifiers were $\mathrm{Pd}\left(\mathrm{NO}_{3}\right)_{2}+\mathrm{Mg}\left(\mathrm{NO}_{3}\right)_{2}$; W/Rh; W+ co-injection of $\mathrm{Pd}\left(\mathrm{NO}_{3}\right)_{2}+\mathrm{Mg}\left(\mathrm{NO}_{3}\right)_{2}$ and 30 samples of ethanol fuel were used for each one. As experimental data were used the results of the addition and recovery tests. The use of the principal component analysis allowed the separation of the different modifiers in function of the recovery range of the analyte. The modifier $\mathrm{W}+$ co-injection of $\mathrm{Pd}\left(\mathrm{NO}_{3}\right)_{2}+\mathrm{Mg}\left(\mathrm{NO}_{3}\right)_{2}$ showed up as the higher positive correlation specie, because shows the higher recovery level, and so, was selected for the development of methodology for direct and simultaneous determination of $\mathrm{Al}, \mathrm{As}, \mathrm{Cu}, \mathrm{Fe}, \mathrm{Mn}$ and $\mathrm{Ni}$ in ethanol fuel by GFAAS.

Keywords: exploratory analysis; chemometrics; PCA; GFAAS; ethanol fuel.

\section{Referências}

[1] A. P. Oliveira, Determinação direta e simultânea de Al, As, $\mathrm{Cu}, \mathrm{Fe}, \mathrm{Mn}, \mathrm{Ni}$, em álcool etílico hidratado combustível por espectrometria de absorção atômica em forno de grafite. 2002. 93f. Dissertação (Mestrado em Química) - Instituto de Química, Universidade Estadual Paulista, São Paulo, 2002.

[2] J. Goldemberg, Energy Policy 24 (1996) 1127.

[3] L. Jolly, Int. Sugar J. 24 (2001), 1127.

[4] B. Welz, M. Sperling, Atomic Absorption Spectrometry, Wiley-VCH, Weinheim, pp.1-56, 1999.

[5] R. E. Bruns, J. F.G. Faigle, Quim. Nova 8 (1985) 84.
[7] K. R. Beebe, B. R. Kowalski, Anal. Chem. 59 (1987) 1007A.

[8] S. Wold, K. Esbensen, P. Geladi, Chemom. Intell. Lab. Syst. 2 (1987) 37.

[9] H. Martens, T. Naes, Multivariate Calibration, John Wiley \& Sons, Chichester, 1993.

[10] M. M. C. Ferreira, A. M. Antunes, M. S. Melgo, P. L. O. Volpe, Quim. Nova 22 (1999) 724.

[11] K. R. Beebe, R. J. Pell, M. B. Seasholtz, Chemometrics: A Practical Guide, John Wiley \& Sons, New York, 1998. [12] A. P. Oliveira, J. A. Gomes Neto, M. Moraes, E. C. Lima, At. Spectrosc. 23(6) (2002) 190. 